\section{Primary Care Diabetes Fellowships: Preparing the Future Primary Care Physician Workforce}

\section{TO THE EDITOR:}

In the June 2020 issue of Family Medicine, ${ }^{1} \mathrm{Dr}$ Saultz described the results of the Professionals Accelerating Clinical and Educational Redesign (PACER) study as "dismal if our shared goal is to produce a primary care workforce." PACER described the conditions influencing collaboration among family medicine, general internal medicine, and general pediatrics residencies. Saultz asks, “... are there really three effective models of primary care residency training at this point in the history of American medicine? PACER suggests not, so maybe it is time to consider more radical ideas."

One radical idea: let's not waste any more time redesigning internal medicine and pediatrics residencies. It doesn't work. Instead, focus on training that has been successful: fellowships. We have demonstrated success in training geriatricians, adolescent specialists, and sports medicine physicians collaboratively with other specialties. One "new" fellowship that will affect a large swath of our patient population and can address some of our workforce needs is diabetes, a true primary care fellowship.

Primary care physicians approach patients from a completely different perspective than specialists. During a keynote address at STFM's 2020 Annual Conference, entitled "Emergence in the Era of Measurement," Kurt Stange and Rebecca Etz presented three simple rules that explain the paradox of primary care. ${ }^{2}$ Primary care physicians recognize and make sense of problems/opportunities, prioritize the most important problems/opportunities, and personalize their approach to generate healing, health, or connection. The three simple rules of specialty care are different, but also important. Specialists identify and classify disease for management, interpret through specialized knowledge, and manage a plan for disease care. Primary care diabetes fellows follow the former three rules (recognize, prioritize and personalize), and then focus on the diagnosis of diabetes from the primary care perspective.

In a paper written collaboratively by family physicians, internists, and endocrinologists, "Primary Care Diabetes Fellowship Programs: Developing National Standards-A White Paper,"3 Jay H. Shubrook, et al describe a successful collaboration in training more than 50 diabetes fellows across six programs to address the care of patients with diabetes. Diabetes affects one in seven Americans and is a major clinical and public health threat. The care of patients with diabetes has rapidly become more complex, and mastering technology while providing comprehensive primary care is a challenge. This paper describes the success of the existing programs in collaboratively training internists and family physicians to become specialists in primary care diabetes. The paper reviews the history of diabetes fellowships, outlines the core competencies of a diabetes fellowship program, and addresses how to build new programs to allow for expansion of primary care diabetes fellowships. Granted, 50 diabetes fellowship graduates will not address the huge need for primary care diabetes specialists in this country, but this white paper provides the blueprint to start 50 new primary care diabetes fellowships!

Diabetes fellowships are great examples of interprofessional collaborative training and practice. Each diabetes fellowship program has physicians, certified diabetic educators, nutritionists, psychologists, and pharmacists caring for patients and teaching collaboratively. In the PACER study, ${ }^{4}$ themes found to positively influence cross-disciplinary collaboration included relationship development, communication of shared goals, alignment with health systems, and professional identity as primary care physicians. Diabetes fellowships embody these themes. Carney et al conclude,

It is clear that primary care plays a vital role in the health of the US population, that innovations in training, especially toward preparing health professionals to be team-based care ready, not just in hospital settings but also in ambulatory settings, are also needed. ${ }^{4}$ 
Diabetes fellowships are a perfect example of a way to meet this need. The fellowships prepare primary care doctors, both internists and family physicians, to provide team-based primary care management of a common chronic condition, while improving quality outcomes across all six quality indicators for diabetes. Diabetes fellowships are one way primary care disciplines can collaborate to achieve the triple aim of health care: improving patient experience and population health while reducing the cost of health care.

doi: 10.22454/FamMed.2020.996607

Lenny Salzberg, MD

Southern Regional AHEC Family Medicine Residency

Fayetteville, NC

Jay Shubrook, DO

Touro University Worldwide

New York, NY

\section{References}

1. Saultz J. False Assumptions. Fam Med. 2020;52(6):393-394.

2. Etz RS, Miller WL, Stange KC. Three simple rules that explain the paradox of primary care. 2020; under review (August 26, 2020 presentation at STFM Virtual Annual Conference).

3. Shubrook JH, Ramirez B, Healy A, et al. Primary Care Diabetes Fellowship Programs: Developing National Standards - A White Paper. Diabetes Care. in press.

4. Carney PA, Thayer EK, Green LA, et al. Conditions influencing collaboration among the primary care disciplines as they prepare the future primary care physician workforce. Fam Med. 2020;52(6):398-407. 